Zeitschriftenartikel:

Begutachtet

Redaktion und Begutachtung:

Nele Heise (iD)

Digital Media \& Communication

Researcher Hamburg

Nils Zurawski (DD

Universität Hamburg

Erhalten: 01. Dezember 2019

Akzeptiert: 29. September 2020

Publiziert: 15. Dezember 2020

Lizenz:

(C) Lisa Leander

Dieses Werk steht unter einer Lizenz

Creative-Commons-Namensnennung 4.0

(CC-BY 4.0) International

(C) (i)

Datenverfügbarkeit:

Alle relevanten Daten befinden sich innerhalb der Veröffentlichung.

Interessenskonfliktstatement:

Die Autor:innen erklären, dass ihre Forschung ohne kommerzielle oder finanzielle Beziehungen durchgeführt wurde, die als potentielle

Interessenskonflikte ausgelegt werden können.

Empfohlene Zitierung:

Leander, L. (2020). Wissenschaft im Gespräch: Wissensvermittlung und -aushandlung in Podcasts.

kommunikation@gesellschaft, 21(2). https://doi.org/10.15460/kommges. 2020.21.2.621

\section{Wissenschaft im Gespräch}

\section{Wissensvermittlung und -aushandlung in Podcasts}

\author{
Lisa Leander ${ }^{\mathrm{a} *}(\mathbb{D})$ \\ ${ }^{a}$ Karlsruhe Institute of Technology \\ *Korrespondenz: lisa.leander@kit.edu
}

\begin{abstract}
Podcasts haben sich als Medium der Wissenschaftskommunikation etabliert. Das zeigt sich einerseits an den Vorlieben des Publikums: Laut den Umfragen des Online-Audio-Monitor rezipieren 70 Prozent der befragten Podcastnutzer:innen „Infosendungen, Wissens- und Lernbeiträge“ (Deck \& Kunow, 2019; vgl. auch Domenichini, 2018; Martens \& Amann, 2007; Schaffer, 2017). Andererseits findet eine Vernetzung der Community statt, wie etwa auf dem Portal wissenschaftspodcasts.de zu sehen ist. Hier kuratieren Produzent:innen das Angebot zu den Themen Wissen und Wissenschaft. Trotz dieser Popularität ist die Vermittlung von Wissenschaft durch Podcasts noch nicht intensiv erforscht, im deutschsprachigen Raum noch weniger als im englischsprachigen. Inhaltliche Analysen konzentrieren sich meist nur auf Teilaspekte wie z.B. Diskussionen in begleitenden Online-Foren (Birch \& Weitkamp, 2010). Im vorliegenden Beitrag soll daher erörtert werden, welche Gestaltungsmöglichkeiten das Medium Podcast speziell für Akteur:innen der Wissenschaftskommunikation bietet. Davon ausgehend wird eine qualitative Untersuchung von Wissenschaftspodcasts mithilfe der Gesprächsanalyse vorgestellt.

Schlagworte: Podcast, Wissenschaftspodcast, Wissenschaftskommunikation, Gesprächsanalyse, Gespräch, Wissensvermittlung, Linguistik
\end{abstract}




\section{Begriffsdefinition: Wissenschaftspodcasts}

Bevor man sich dem Feld, Wissenschaftspodcasts' zuwendet, muss zunächst geklärt werden, was darunter zu verstehen ist. MacKenzie (2019) hat eine Analyse von rund 1.000 englischsprachigen science podcasts durchgeführt und grenzt seine Auswahl folgendermaßen ein: Um als science podcast zu gelten, "a podcast must be on covering scientific topics, for example, scientific research, science news, scientific careers, scientific seminars, lectures, or similar" (MacKenzie, 2019, S. 13). Birch \& Weitkamp (2010) lassen ein ähnliches Verständnis des Begriffs erkennen und schließen explizit pseudowissenschaftliche und nicht-wissenschaftliche Themen aus.

Laut MacKenzie (2019) müssen science podcasts also nicht zwangsläufig fachwissenschaftliche Themen behandeln, sie sollten jedoch einen Bezug zum Forschungsbetrieb aufweisen. Unterstützt wird dieser Ansatz von Hellermann (2015), der Wissenschaft als Dispositiv beschreibt. Damit bezieht er sich zwar auf Wissenschaftspopularisierung im Fernsehen, seine Definition ist aber ebenso auf andere Medien anwendbar. Das Dispositiv Wissenschaft besteht laut Hellermann aus dem wissenschaftlichen Diskurs als Kernelement in Verbindung mit weiteren Teilgebieten wie Forschung, Lehre, Infrastruktur und Administration. Entsprechend umfasse die Wissenschaftskommunikation neben der Vermittlung des wissenschaftlichen Wissens jegliche Kommunikation zu allen soeben genannten Bereichen der Wissenschaft. Damit grenzt er wissenschaftliches Wissen von anderen Arten des Wissens ab, wie beispielsweise praktisches Wissen oder Alltagswissen, die nicht rational überprüfbar sein müssen (Hellermann, 2015, S. 136ff).

Mit Hellermanns Definition lässt sich ausreichend genau beschreiben, auf welche Themen ein Podcast über Wissenschaft Bezug nehmen kann. Zuletzt sei darauf hingewiesen, dass der im Deutschen übliche Begriff ,Wissenschaftspodcast' breiter gefasst ist als science podcast, da er neben Natur- und Technikwissenschaften (science and engineering) auch Geistes-, Sozial- und Kulturwissenschaften (arts and humanities) einschließt (vgl. Birch \& Weitkamp, 2010; MacKenzie, 2019). Wenn also in diesem Beitrag Wissenschaftspodcasts diskutiert und analysiert werden, ist das Feld, auf das Bezug genommen wird, größer als in den meisten englischsprachigen Untersuchungen.

\section{Podcasts als Instrument der Wissenschaftskommunikation}

Bei der Forschung zu Wissenschaft in Podcasts fällt auf, dass ein Schwerpunkt auf dem Einsatz für Lehre und Lernen liegt (vgl. Tynan \& Colbran, 2006; Bruce \& Lin, 2009; Teckchandani \& Obstfeld, 2017). Hier werden Aspekte wie Wissenserwerb, Motivation und Lernverhalten betrachtet. Welche Bedeutung Podcasts hingegen für die Wissenschaftskommunikation haben, ist bislang wenig untersucht (für eine Definition des Feldes siehe z.B. Schäfer, Kristiansen \& Bonfadelli (2015)). Dies könnte daran liegen, dass Zielgruppen und 
Zielsetzungen der einzelnen Formate nicht so klar zu fassen sind, wie es bei Podcasts als Lernmedium der Fall ist. Viele Vorteile, die Podcasts als Kommunikationskanal zugeschrieben werden (keine Längenbegrenzung, geeignet für sogenannte Nischenthemen etc.), gelten nicht nur für die Darstellung von Wissenschaft, sondern ebenso für andere Themen. Daher stellen sich die Fragen: Was sind spezifische, verbindende Elemente von Wissenschaftspodcasts? Inwiefern prägen diese die Wissenschaftskommunikation, die durch das Medium stattfindet?

In der bereits erwähnten Studie von MacKenzie (2019) zeigt sich, dass im Bereich Podcasting ein breites Spektrum von Akteur:innen der Wissenschaftskommunikation aktiv ist: Neben Wissenschaftler:innen selbst zählen dazu Medienredaktionen, PR-Abteilungen sowie Personen, die weder in der Forschung noch journalistisch tätig sind und dennoch am Dialog über wissenschaftliche Themen teilnehmen. Etwa ein Drittel der Podcasts in MacKenzies Sample stammt von unabhängigen Anbieter:innen, der übrige Anteil von knapp zwei Dritteln steht in Zusammenhang mit Organisationen. Dabei handelt es sich u.a. um professional organisations, Universitäten, Medienverlage, andere Forschungsinstitutionen oder Podcast-Netzwerke, in denen sich mehrere Produzierende zusammenschließen (MacKenzie, 2019, S. 8).

Es zeichnet sich ein Bild ab, das für andere Themenbereiche ebenso gültig ist: Podcasts werden häufig als User-Generated-Content und GraswurzelKommunikation charakterisiert (vgl. Domenichini, 2018) und waren vor allem in den Anfängen mit der Idee des aktiven prosumer verbunden (vgl. Markman, 2012; Lüders, 2008). Die Hoffnung, dass viele Hörende selbst zu Produzierenden werden, hat sich nur teilweise erfüllt, da institutionelle Anbieter:innen über deutlich bessere Verbreitungsstrukturen und Finanzierungsquellen verfügen. Dadurch können sie einfacher eine große Reichweite generieren und sind mit ihren Angeboten entsprechend erfolgreich (vgl. Swiatek, 2018; Bottomley, 2015). Doch eine Stärke des Mediums besteht darin, dass es über den sogenannten long tail funktioniert - während einige wenige Podcast-Formate ein Massenpublikum erreichen, hat der Großteil der Produzierenden eine verhältnismäßig kleine Hörerschaft (Markman \& Sawyer, 2014). Da Podcasts kostengünstig zu produzieren und zu verbreiten sind, lohnt es sich selbst für unabhängige Podcaster:innen, ein sehr speziell interessiertes Publikum anzusprechen. Die Diversität auf Seiten der Anbieter:innen resultiert folglich in einer größeren Vielfalt der Inhalte (ebd.).

Dies ist vor allem interessant in Hinblick auf Gespräche als Beitragsformat. Schriftliche Medien enthalten - abgesehen von Interviews - monologische Texte, in denen andere Stimmen als die des Autors oder der Autorin nur als Zitate vorkommen. In audiovisuellen Medien wie Radio oder Fernsehen hingegen können z.B. Interviewgast und Moderator:in direkt miteinander interagieren und aufeinander reagieren (Burger \& Luginbühl, 2014). Bei der Vermittlung von Fachwissen treten die beteiligten Akteur:innen dabei in der Regel als Expert:in, Lai:in oder Moderator:in auf (ebd.). Solche Gesprächsrollen wurden für die Rundfunkmedien bereits ausführlich betrachtet (vgl. Burger, 
1991; Clayman, o. J.; Hutchby, 2007).

Podcasts schlagen nun eine Brücke zwischen dem partizipativen Charakter des Web 2.o und den Möglichkeiten elektronischer, audiovisueller Medien, die eine direkte Interaktion im Gespräch zulassen. Für Wissenschaftspodcasts resultiert daraus, dass die oben genannten typischen Rollen von anderen Akteur:innen eingenommen werden bzw. die gleichen Akteur:innen in unterschiedlichen Gesprächskonstellationen zusammenkommen (vgl. Jarrett, 2009). Bislang wurde in einzelnen Fallanalysen u.a. diskutiert, wie wissenschaftliche Amateur:innen, die Hosts von Wissenschaftspodcasts sind, mit ihrem fehlenden fachlichen Hintergrund umgehen (Barrios-O'Neill, 2018; Salvati, 2015). Dies berührt bereits die Frage, wer sich als Expert:in positioniert und auf welche Weise dies geschieht. Es ist ebenso interessant zu betrachten, wer die Moderation übernimmt, wenn z.B. in einem Podcast ausschließlich Wissenschaftler:innen auftreten.

\section{Gegenstand der Untersuchung: Gespräche in Wissenschaftspodcasts}

Die folgende Analyse konzentriert sich daher auf Gespräche, die in Podcasts zum Zwecke der Wissenschaftsvermittlung stattfinden. Anders als bei Magazinsendungen mit eingespielten O-Tönen oder monologischen Formaten (vgl. Chamberlin, 2019) findet in der direkten Interaktion eine Aushandlung und Zuweisung von Wissen statt, die mit den oben erwähnten Rollen zusammenhängt (vgl. Deppermann, 2018). Gespräche über Wissenschaft sind zwar auch in anderen Online-Medien möglich wie etwa in Web-Videos. Jedoch sind solche Videos im Durchschnitt meist kürzer in der Dauer (Welbourne \& Grant, 2016) und enthalten zusätzlich die visuelle Ebene. Audio-Podcasts sind hingegen auf das Gesagte und die darin hergestellten Sinnzusammenhänge reduziert. Daher ist die hier vorgestellte Untersuchung auf den Bereich Audio beschränkt, die Sparte der Video-Podcasts wird nicht berücksichtigt.

Die Ausführungen geben Einblicke in ein laufendes Dissertationsprojekt und sind daher als erste Ansatzpunkte zu verstehen. Neben den genannten Akteur:innen und Rollenkonstellationen gibt es weitere Faktoren, die Einfluss darauf haben könnten, wie Gespräche in Podcasts organisiert sind. Dazu gehören z.B. der serielle Charakter von Podcasts (ermöglicht Querverweise zwischen Folgen) oder die zeitversetzte Nutzung (Publikum kann nicht live angesprochen werden, vgl. Jarrett (2009)). Diese Aspekte werden an dieser Stelle vorerst ausgespart, da sie keine spezifischen Merkmale von Wissenschaftspodcasts sind. Im Dissertationsprojekt finden sie hingegen Beachtung.

Die hier präsentierten Podcasts sind einem deutlich größeren Sample aus deutschsprachigen Audiopodcasts entnommen, die auf den Portalen pan- 
optikum.io ${ }^{1}$ und fyyd.de ${ }^{2}$ veröffentlicht wurden. Auf beiden Portalen können Podcasts nach Themen und Sprache gefiltert werden und die Anzahl der aufgeführten Formate ist ausreichend groß für die Untersuchung. Das Angebot wurde gesichtet und nach der oben genannten Definition für Wissenschaftspodcasts selektiert. Außerdem erfolgte eine Kategorisierung anhand von Themen, Produktionsbedingungen, Beitragsformen etc. Für das finale Sample sind nur Podcasts von Bedeutung, die Gespräche - also eine direkte sprachliche Interaktion zwischen den Teilnehmenden - enthalten. Ebenfalls ausgeschlossen wurden Podcasts, deren letzte Folge länger als ein Jahr vor Ziehung des Samples im September 2019 zurückliegt. Dies geschah deshalb, weil sich die inhaltliche Gestaltung von Podcasts durch verbesserte Aufnahmetechniken, Trends o.Ä. über die Jahre verändert haben könnte. Darauf zurückzuführende Unterschiede sollten ausgeschlossen werden. Abschließend wurden die jeweils aktuellsten Folgen von sieben Podcasts ausgewählt. In diesem Beitrag werden fünf davon präsentiert:

Tabelle 1: Ausgewählte Folgen deutschsprachiger Wissenschaftspodcasts Quelle: Eigene Darstellung (Anmerkung: Vor- und Zunamen sind wie in der Beschreibung von Podcast und Folge angegeben)

\begin{tabular}{|c|c|c|c|c|}
\hline Podcast & Produktion & Themenfeld & Folge & Teilnehmende \\
\hline THINK \& DO & $\begin{array}{l}\text { Produziert vom } \\
\text { Stifterverband für die } \\
\text { Deutsche Wissenschaft }\end{array}$ & Verschiedenes & $\begin{array}{l}\text { "T\&D08: } \\
\text { \#Lecturesforfuture“, } \\
\text { veröffentlicht am } \\
\text { 12.06.2019 }\end{array}$ & $\begin{array}{l}\text { Michael Sonnabend } \\
\text { (Host), Joachim } \\
\text { Fensterle (Gast) }\end{array}$ \\
\hline Jung und Freudlos & $\begin{array}{l}\text { Unterstützt und he } \\
\text { rausgegeben durch die } \\
\text { Universität Freiburg }\end{array}$ & Psychologie & $\begin{array}{l}\text { „Kinder und } \\
\text { Jugendpsychiatrie“, } \\
\text { veröffentlicht am } \\
\text { 20.09.2019 }\end{array}$ & $\begin{array}{l}\text { Ismene, Moritz, } \\
\text { Sebastian (Hosts), } \\
\text { Sebastian Herr (Gast) }\end{array}$ \\
\hline $\begin{array}{l}\text { Transformatorische } \\
\text { Bildung }\end{array}$ & Privat produziert & $\begin{array}{l}\text { Bildungs- und } \\
\text { Erziehungs- } \\
\text { wissenschaft }\end{array}$ & $\begin{array}{l}\text { „Anrufungen von } \\
\text { LehrerInnen mit } \\
\text { Migrationshinter- } \\
\text { grund“, veröffentlicht } \\
\text { am 02.09.2019 }\end{array}$ & $\begin{array}{l}\text { Tim Schmidt (Host), } \\
\text { Sirin (Gast) }\end{array}$ \\
\hline $\begin{array}{l}\text { Ach? - Triumvirat für } \\
\text { historisch inspirierte } \\
\text { Humorvermittlung }\end{array}$ & Privat produziert & Geschichte & $\begin{array}{l}\text { „33 - Die Tortour der } 1 . \\
\text { Tour de France“, } \\
\text { veröffentlicht am } \\
30.07 .2019\end{array}$ & $\begin{array}{l}\text { Dominik, Jürgen, } \\
\text { Philipp (Hosts) }\end{array}$ \\
\hline Sternbildung & $\begin{array}{l}\text { Auftragsproduktion } \\
\text { von Spektrum der } \\
\text { Wissenschaft }\end{array}$ & Astronomie & $\begin{array}{l}\text { „Löwe - ein brauner } \\
\text { Zwerg“, veröffentlicht } \\
\text { am 01.06.2019 }\end{array}$ & $\begin{array}{l}\text { Florian Freistetter, } \\
\text { Holger Klein (Hosts) }\end{array}$ \\
\hline
\end{tabular}

Die Podcasts sollen ein möglichst breites Spektrum an Themen, Anzahl der teilnehmenden Gesprächspartner:innen und Produktionsbedingungen abdecken. Beispielsweise sind die Podcasts THINK \& $D O^{3}$ und Jung und Freudlos ${ }^{4}$ beide dem Feld der Public Relations zuzuordnen, allerdings werden sie unterschiedlich produziert: THINK \& DO ist Teil der zentralen Kommunikation des Stifterverbands und wird vom Leiter der Öffentlichkeitsarbeit präsentiert, während der Podcast Jung und Freudlos in den Händen einer Gruppe von Assistenzärzt:innen bzw. Student:innen liegt. Hier treten also verschiedene Ak-

\footnotetext{
1 https://panoptikum.io/ (Zugriff am 01.11.2019).

2 https://fyyd.de/ (Zugriff am 01.11.2019).

3 https://thinkanddo.podigee.io/ (Zugriff am 01.11.2019).

4 https://juf.podigee.io/ (Zugriff am 01.11.2019).
} 
teur:innen auf, deren mehr oder weniger starke Anbindung an die Organisationskommunikation möglicherweise beeinflusst, wie sie Inhalte auswählen und darstellen.

In manchen der vorgestellten Podcasts sprechen nur die sogenannten Hosts miteinander, die zur festen Besetzung des Podcasts gehören, in anderen werden wechselnde Gäste eingeladen. Ebenfalls ein Kriterium für die Untersuchung ist, ob es sich bei Hosts und Gästen um Wissenschaftler:innen, professionelle Journalist:innen oder PR-Mitarbeiter:innen oder nichts von beidem handelt. Das ist in der Tabelle noch nicht erkennbar, wird aber in den folgenden Abschnitten thematisiert.

\section{Einführung und Methodik: Gesprächsanalyse}

Die Ausschnitte der Podcast-Folgen werden mit den Mitteln der Gesprächsanalyse nach Harvey Sacks untersucht. Diese dient zur Beschreibung von „recorded, naturally occuring talk-in-interaction" (Hutchby \& Wooffitt, 2008, S. 12), also der Interaktion der Teilnehmenden in einem natürlichen Gespräch im Gegensetz etwa zu fiktiven Dialogen in einem Theaterstück (Henne \& Rehbock, 1979). In der Gesprächsanalyse wird davon ausgegangen, dass die Akteur:innen sich gegenseitig anzeigen, welche Bedeutung sie ihren Äußerungen zuweisen, und wie diese zu verstehen sind (Deppermann, 2008). Der Fokus liegt deshalb darauf, wie die Sprechenden aufeinander reagieren und das Gespräch bewusst lenken. Ebenfalls von Interesse ist, an welchen Konventionen, unausgesprochenen Regeln oder Rollenbildern sich die beteiligten Personen orientieren (Hutchby \& Wooffitt, 2008).

Die Methode der Gesprächsanalyse wurde bereits auf Podcasts angewandt vgl. Jarrett (2009); Norman (2018)], jedoch nicht in Hinblick auf die Vermittlung von Wissen und Wissenschaft. Dies ist insofern überraschend, da Gespräche zur Informationsvermittlung in Rundfunkmedien - zum Beispiel durch Nachrichteninterviews - bereits ausführlich analysiert wurden, wie das nächste Kapitel zeigen wird. Bis hierhin sollte klar geworden sein, dass Podcasts ein sehr breites Spektrum an Audio-Produktionen abdecken, entsprechend groß ist die Anzahl an Akteur:innen der Wissenschaftskommunikation, die in den Podcasts mitwirken.

Die Analyse der ausgewählten Podcast-Folgen soll daher zeigen, welche Akteur:innen hier aufeinandertreffen. Darüber hinaus wird beschrieben, wie sie das Gespräch organisieren, um wissenschaftliche Inhalte an ihr Publikum zu vermitteln, und wie sie ihr eigenes Wissen und Verständnis einbringen. Ein Fokus liegt dabei auf dem Verständnis der eigenen Rolle, etwa die als Expert:in oder Lai:in bezogen auf das präsentierte Thema.

Als Einführung dient zunächst ein Überblick zu Gesprächen in den Medien und den Rollen, die darin vorkommen. Anschließend folgt eine kurze Erklärung zur Methode der Gesprächsanalyse. 


\section{1 Überblick: Analyse von Mediengesprächen}

Gespräche in den Medien - sogenannte Mediengespräche - unterscheiden sich zwar grundlegend von Gesprächen in Alltagssituationen (Hutchby, 2007). Dennoch ist es laut Hutchby nicht nur möglich, sondern hilfreich, beide Arten der interpersonellen Kommunikation zu vergleichen. Denn erst durch die Kontrastierung mit Alltagsgesprächen trete hervor, wie die Situation, die durch das Medium entsteht, Einfluss nimmt auf den Verlauf der Konversation (ebd., S. 17).

Hutchby beschreibt Gespräche in den Rundfunkmedien (broadcast talk) als "specific type of institutional discourse because it is directed at an 'overhearing' audience separated from the talk's site of production by space and also, frequently, by time" (ebd., S. 18, Herv.i.O.). Sie sind damit in einen bestimmten Kontext eingeordnet, andere settings wären beispielsweise Schule, Arbeitsplatz, Gerichtssaal oder Arztpraxis (ebd., S. 24). Aus Sicht der Gesprächsanalyse handelt es sich dabei jedoch nicht um festgelegte soziale Rahmen, auf welche die beteiligten Akteur:innen lediglich reagieren (ebd.). Stattdessen wird aus dem Verhalten der Akteur:innen darauf geschlossen, an welchen Rahmenbedingungen und teils unausgesprochenen sozialen Regeln sie sich orientieren und dadurch den Kontext konstruieren (vgl. Hutchby, 2007, S. 18).

\subsection{Expert:innen in den Medien und weitere Rollen}

Als Nächstes sollen die möglichen Rollen in Gesprächen mit Fokus auf das Thema Wissenschaft näher vorgestellt werden. Eine sehr wichtige Position nehmen dabei die Expert:innen ein. Aus Sicht der Medien werden sie darüber definiert, dass sie über spezielles Wissen sowie Problemlösungskompetenz verfügen - im Gegensatz zum sogenannten Laien oder der Laiin, dem oder der eben dieses Wissen und die Kompetenz fehlen (Burger \& Luginbühl, 2014). Beide Rollen existieren nur in Relation zueinander: Es kann nur dann von einem Experten oder einer Expertin gesprochen werden, wenn diese Person von anderen aufgrund ihres Wissens konsultiert wird. Ebenso kann nur dann von dem Laien oder der Laiin gesprochen werden, wenn er oder sie eben diesen Rat einholt (Nölleke, 2013; Peters, 1994).

Im Gegensatz dazu wird die Rolle der Moderator:innen durch ihre Funktion im Gespräch bestimmt. Sie sind meist die Gastgeber:innen einer Sendung und können als Stellvertreter:innen des Publikums fungieren, indem sie etwa Verständnisfragen an die Expert:innen richten (Burger, 1991; Burger \& Luginbühl, 2014).

Burger \& Luginbühl (2014) beschreiben eine interessante Situation in einer Gesundheitssendung im Fernsehen, in der die Moderatorin ebenfalls Medizinerin ist. Das ist daran erkennbar, dass sie gelegentlich selbst Fachwörter benutzt oder eine Erklärung liefert (Burger \& Luginbühl, 2014, S. 375ff). Gleichzeitig bemüht sie sich in ihrer eigentlichen Rolle der Moderatorin zu bleiben, 
indem sie ihr Wissen zurückhält und Fragen aus Sicht von wissenschaftlichen Lai:innen stellt (ebd.). Ihre Rolle im Gespräch steht damit über der, die sie von ihrem Wissensstand her einnehmen könnte. Bei dem Moderator oder der Moderatorin handelt es sich daher ebenfalls um eine zugewiesene Rolle, die über den Umgang mit den anderen Gesprächspartner:innen definiert ist.

Ein anderes Beispiel dazu stammt aus einer TV-Dokumentation. Der Moderator übernimmt im Gespräch mit einem Experten die Rolle des Fragenden, während er im nächsten Abschnitt der Sendung selbst Fachinhalte referiert (Chovanec, 2016). Die zweite Position nimmt er jedoch nur ein, als er sich nicht mehr in Interaktion mit dem Experten befindet. Der Rollenwechsel findet damit nicht spontan während des Gesprächs statt, wie im vorigen Beispiel, sondern wird im Verlauf der Sendung bewusst inszeniert.

Goffman (1981) führt eine weitere Ebene zur Rolle der Moderation ein, in Bezug auf Sprecher:innen (speaker) im Radio. Er weist darauf hin, dass der Begriff verschiedene Bedeutungen haben kann: animator, author oder principal (Goffman, 1981, S. 226). Animator steht für die Person, die den Text spricht, author für die, die ihn geschrieben oder zusammengestellt hat, und principal ist die Institution, welche die im Text präsentierte Meinung vertritt (ebd.). Der oder die Sprechende kann dabei gleichzeitig die Funktion von animator, author und principal einnehmen oder während des Gesprächs von einer oder mehreren dieser Positionen abweichen (Hutchby, 2007, S. 128).

Zusammengefasst sind drei Ebenen zu erkennen, auf denen eine Rollenverteilung in Mediengesprächen zu Wissenschaft und Information stattfinden kann:

- die Rolle aufgrund des eingebrachten Wissensstandes und dessen Anwendung (Expert:in, Lai:in)

- die Rolle aufgrund der Funktion im Gespräch (Gastgeber:in, Teilnehmende:r bzw. Gast, Vertreter:in des Publikums etc.)

- die Rolle bezogen auf die Inhalte der Aussagen (author, animator, principal)

\section{Analyse ausgewählter Podcastfolgen}

\subsection{Methodische Vorbemerkungen}

Die folgenden Ausschnitte wurden nach der Konvention cGAT mit den Programm FOLKER transkribiert und liegen in der aktuellen Arbeitsphase als Minimaltranskript vor. Dieses Transkript stellt die erste Stufe der Analyse dar und wird im weiteren Vorgehen zum sogenannten Basis- oder sogar Feintranskript erweitert. Jede Stufe enthält zusätzliche Informationen zur Prosodie (Betonungen, Sprechtempo, Rhythmus etc., (Selting, Auer \& Barth-Weingarten, 2009)). Im Dissertationsprojekt ist außerdem geplant, 
ergänzend zur Gesprächsanalyse qualitative Leifadeninterviews mit den Hosts der Podcasts durchzuführen und auszuwerten.

Das hier gezeigte Minimaltranskript gibt lediglich das wörtlich Gesagte wieder sowie einige weitere Signale und Informationen:

+++ unverständliche Passage

(solche) vermuteter Wortlaut, nicht sicher rekonstruierbar

((lacht)) non-verbale Äußerungen

${ }^{\circ} \mathrm{h} /{ }^{\circ} \mathrm{hh}$ hörbares Einatmen

(0.5) Sprechpause mit Angabe der Dauer in Sekunden

[ ] [ ] überlappende Gesprächsanteile oder gleichzeitiges Ansetzen (simultan gesprochene Passagen werden untereinander gruppiert)

Der Schwerpunkt der Analyse liegt auf den eben vorgestellten Rollen. Es ist die Frage zu beantworten, inwiefern sie sich auch in Wissenschaftspodcasts wiederfinden und ob sie von den beteiligten Akteur:innen in den unterschiedlichen Gesprächskonstellationen variiert oder neuinterpretiert werden.

\subsection{Grundlagen der Gesprächsorganisation}

Eine typische Form des Mediengesprächs, das der Vermittlung von Wissen und Informationen dient, ist das Nachrichteninterview. Es folgt klaren Regeln, an denen sich die Gesprächspartner:innen orientieren, und anhand derer die Zuhörenden sofort erkennen, um welche Art der Interaktion es sich handelt (Heritage \& Clayman, 2010, S. 216): Der Ablauf ist dadurch gekennzeichnet, dass der/die Interviewende die Fragen stellt, während der/die Interviewte antwortet. Dadurch wird das sogenannte turn taking bestimmt. Der Begriff beschreibt, wie der Sprecher:innenwechsel organisiert ist, also an welchen Stellen die Gesprächspartner:innen in der gemeinsamen Interaktion das Wort ergreifen. Jeder ,Turn' entspricht vereinfacht gesagt einem Redebeitrag (ebd.). Außerdem nimmt der oder die Interviewende - im Rundfunk in der Regel ein:e Journalist:in - typischerweise eine neutrale Position ein und vertritt keine persönlichen Ansichten oder die der eigenen Organisation (ebd.).

Gesprächsmuster des Nachrichteninterviews sind auch in den oben genannten Podcast-Folgen erkennbar. In der Folge von THINK \& DO (Sonnabend 2019) spricht Michael Sonnabend (S), Leiter der Öffentlichkeitsarbeit und internen Kommunikation beim Stifterverband, mit Joachim Fensterle (F) von der Hochschule Rhein-Waal über dessen Engagement bei der Initiative „Lessons for Future": 


\begin{tabular}{|c|c|c|}
\hline 0002 & & ${ }^{\circ} \mathrm{h}$ ähm also so ist das im prinzip entstanden \\
\hline 0003 & & [ja] \\
\hline 0004 & $\mathrm{~s}$ & [ok] \\
\hline 0005 & & ähm die scientists for future ähm \\
\hline 0006 & & $(0.62)$ \\
\hline 0007 & & machen da auch mit oder_s in Kooperation mit denen oder \\
\hline 0008 & & +++ \\
\hline 0009 & $\mathrm{~F}$ & [genau also wir ham ] \\
\hline 0010 & & die scientists for future die ham ja ähm eine ähm im prinzip so ne so nen aufruf äh wo \\
\hline 0011 & & $\begin{array}{l}\text { ich will jetzt nicht lügen hab so (grau) rund siebenundzwanzigtausend Wissenschaftler } \\
\text { unterschrieben haben }\end{array}$ \\
\hline 0012 & & $\begin{array}{l}\text { wo sie in dem aufruf in prinzip auch ähm den äh zum zum äh handeln bezüglich } \\
\text { klimawandel aufgefordert haben }\end{array}$ \\
\hline
\end{tabular}

Bei Zeile 01 signalisiert Fensterle das Ende seines Turns mit einer zusammenfassenden Bemerkung ( ,also so ist das im Prinzip entstanden") und gibt Sonnabend so das Signal, dass er bereit ist für die nächste Frage (vgl. Hutchby \& Wooffitt, 2008, S. 144ff). Obwohl dieser die Frage in Zeile 07 nicht zu Ende formuliert, hat Fensterle sie gut genug verstanden, um den Turn wieder zu übernehmen und zur nächsten Ausführung anzusetzen. Die Rollen des Fragenden und des Antwortenden sind hier klar verteilt und beide Gesprächsteilnehmer richten ihr Verhalten danach aus.

Der Verlauf des Gesprächs ist damit ähnlich dem von Nachrichteninterviews in journalistischen Medien. Allerdings nimmt Sonnabend als Mitarbeiter des Stifterverbands nicht die Position eines neutralen Berichterstatters ein. Das tritt an einer anderen Stelle hervor:

F

ich glaub wenn ich die postleitzahlenliste mir durchgucke wir haben wirklich aus jedem aus jedem postleitzahlenbereich mindestens eine veranstaltung oder mehrere also das ist schon so dass

[das]

$[\mathrm{mh}]$ 


\begin{tabular}{|c|c|c|}
\hline 0006 & S & {$[\mathrm{mh}]$} \\
\hline 0007 & & $\begin{array}{l}\text { 'hh muss sich ja wahrscheinlich dann auch erstmal en bissel rumsprechen ne ähm also sie } \\
\text { sind ja jetzt glaub ich ein paar wochen sozusagen mit dem aufruf in der welt ähm vielleicht } \\
\text { hilft ja auch dieses gespräch noch }\end{array}$ \\
\hline 0008 & & [en bisschen ((lacht))] \\
\hline 0009 & $\mathrm{~F}$ & {$[(($ lacht $))]$} \\
\hline 0010 & S & $\begin{array}{l}\text { die idee noch en bisschen weiterzutragen also wir beim stifterverband sind alle sehr } \\
\text { begeistert äh von diesen }{ }^{\circ} \mathrm{hh} \text { projekt und unterstützen das natürlich dann auch gerne }\end{array}$ \\
\hline 0011 & $\mathrm{~F}$ & ja das freut mich sehr \\
\hline
\end{tabular}

Sonnabend stellt ab Zeile 07 nicht die nächste Frage, sondern macht eine Bemerkung im Namen seiner Organisation. Er drückt die Unterstützung des Stifterverbands für die „Lessons for Future“ aus, die schon dadurch demonstriert wird, dass Fensterle als Gast für den Podcast eingeladen wurde. Fensterle gibt den Turn zwar nicht so eindeutig ab wie im Beispiel davor, zeigt aber durch das einstimmende Lachen in Zeile 09, dass er die Abweichung vom bisherigen Frage-Antwort-Muster erkennt und akzeptiert. Mit seinem Schlusskommentar begrüßt er sie sogar ausdrücklich. Er ist in diesem Gespräch der Experte für das Thema, wobei es nicht um seine eigene Forschung als Wissenschaftler, sondern um sein gesellschaftliches Engagement geht. Ebenso wie Sonnabend ist er gleichzeitig Advokat für seine Organisation bzw. Initiative.

Eine solche Abweichung von der (neutralen) Rolle der Moderation wurde ähnlich bereits für Gespräche im freien Radio beschrieben als Kennzeichen eines alternativen Hörfunks (Pinseler, 2001). Hier teilen Interviewende ebenfalls den Standpunkt ihrer Gäste. Das Phänomen ist daher nicht neu. Es bleibt festzuhalten, dass durch Podcasts, die u.a. für die Öffentlichkeitsarbeit als Kommunikationskanal genutzt werden können, neue Akteur:innen hinzukommen, die dieses Verhalten im Gespräch zeigen. Im alternativen Hörfunk ist es akzeptiert, dass die Moderator:innen eigene Meinungen oder Sympathien ausdrücken, die ggf. auch der gesellschaftlichen oder politischen Ausrichtung des Senders entsprechen. Im Podcast THINK \& DO kommt diese Rolle des principal sogar noch stärker zum Tragen, da Sonnabend als Pressesprecher des Stifterverbands auftritt und agiert.

\subsection{Expert:innen und Ko-Expert:innen}

Oben wurde vorgestellt, wie ein Gespräch zwischen Moderator:in und Expert:in häufig abläuft: Es ist zweifelsfrei erkennbar, wer die Informationen vermittelt. Für Wissenschaftspodcasts stellt sich die Frage, ob Wissenschaftler:innen, die als Hosts mit Gästen sprechen, ebenfalls als Interviewende auftreten und ihr Expertenwissen bewusst zurückhalten. Als Beispiel soll der 
Podcast Jung und Freudlos dienen. Gast der exemplarischen Folge (Hermann et al. 2019) ist Sebastian Herr (H), der über sein Fachgebiet der Kinder- und Jugendpsychiatrie am Universitätsklinikum Freiburg berichtet. Der Podcast hat drei Hosts, zwei davon treten im ausgewählten Ausschnitt auf: Moritz (M), Medizinstudent, und Sebastian (S), Assistenzarzt für Psychiatrie, die beide ebenfalls in Freiburg arbeiten bzw. studieren. ${ }^{5}$

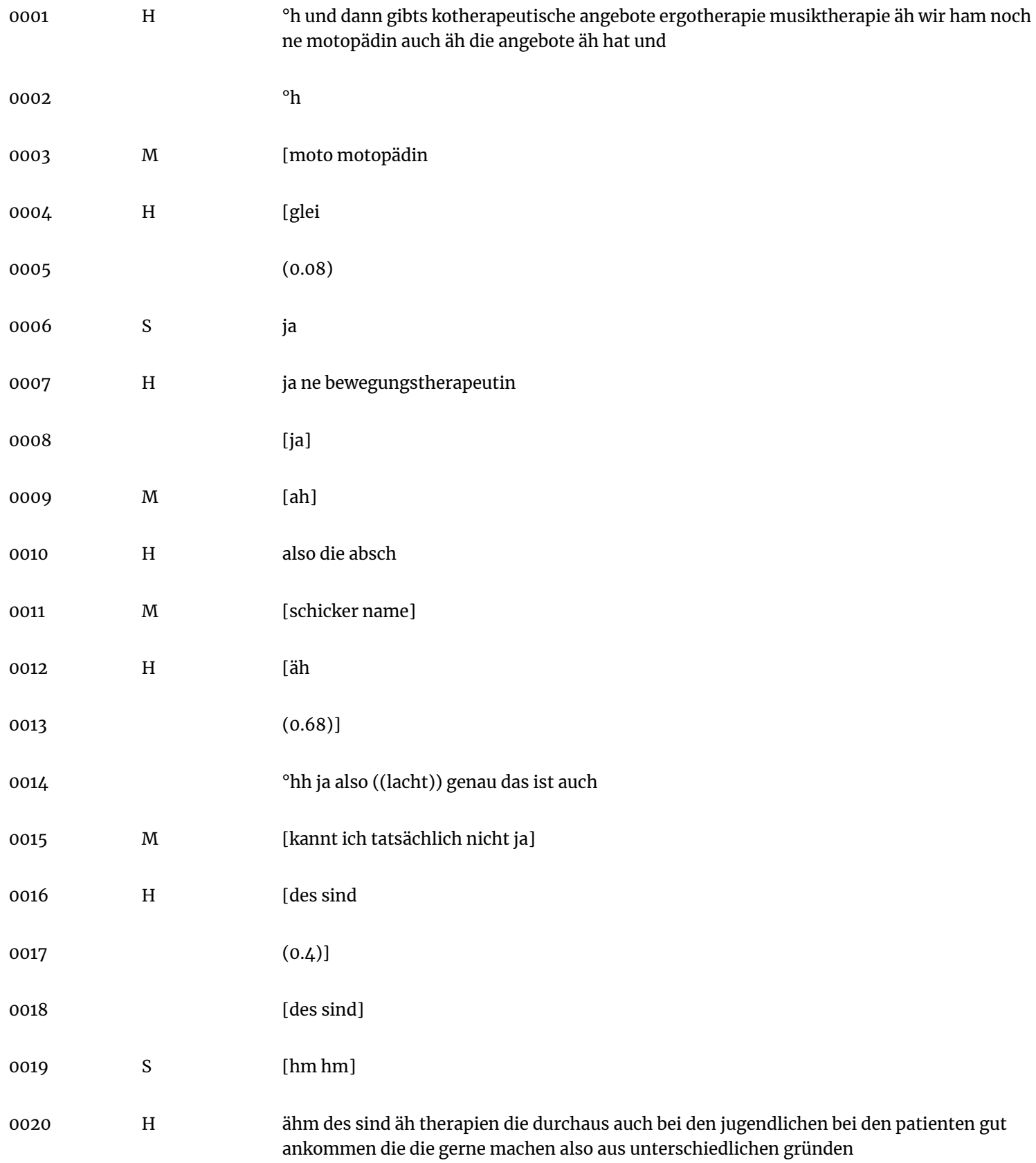

5 Aufgrund der Ähnlichkeit der Stimmen der beiden männlichen Hosts (Moritz und Sebastian) fällt es schwer, sie bei diesem Ausschnitt zweifelsfrei zuzuordnen. Für die weitere Interpretation ist das jedoch nicht von Bedeutung. 
Herr als Experte benutzt den Fachbegriff „Motopädin“ entweder aus Gewohnheit oder unter der Annahme, er sei bekannt. Das ist nicht der Fall, wie die Einwürfe von Moritz und Sebastian ab Zeile 03 zeigen. Herr nimmt deshalb eine sogenannte ,Reparatur' vor (vgl. Kitzinger, 2012), indem er den leichter verständlichen Begriff „Bewegungstherapeutin“ wählt. Die Reparatur hat im Gespräch die Funktion, das Verstehen zwischen den Gesprächspartner:innen wiederherzustellen. Sebastian und Moritz zeigen an, dass ihnen nun die Bedeutung klar ist. Auffällig ist die Bemerkung bei Zeile 15: Moritz lässt erkennen, dass er die Nachfrage nicht nur im Interesse der Zuhörenden gestellt hat, sondern die Bedeutung des Begriffs selbst nicht wusste. Gleichzeitig drückt er seine Überraschung über eben diesen Umstand aus, denn als Medizinstudent hätte ihm das Fachwort geläufig sein können.

Moritz ist also während des Gesprächs Fragestellender und zeigt im gleichen Zuge seine Expertise an. Obwohl Herr als Facharzt auf dem Gebiet der Kinder- und Jugendpsychiatrie in den Podcast eingeladen wurde, verfügen die drei Hosts zumindest über einen ähnlichen Erfahrungsschatz. Das trifft auf Sebastian und Moritz ebenso zu wie auf ihre Kollegin Ismene (I), ebenfalls Assistenzärztin:

\begin{tabular}{|c|c|c|}
\hline 0001 & $\mathrm{H}$ & $\begin{array}{l}\text { das ist durchaus im bewusstsein dass es die kinderpsychatrie auch gibt auch bei den } \\
\text { kinderärzten und äh da gibts eigentlich ja also ich hab das gefühl dass die }\end{array}$ \\
\hline 0002 & & $(($ schluckt $))$ \\
\hline 0003 & & $\begin{array}{l}\text { durchaus auch patienten zuweisen dass die da als erste stelle beim kinderarzt sind und } \\
\text { dass dann weitervermittelt wird }\end{array}$ \\
\hline 0004 & S & {$[\mathrm{mhm}]$} \\
\hline 0005 & I & {$[\mathrm{hm}]$} \\
\hline 0006 & M & ok also en bisschen wie bei den erwachsenen in klein diesmal \\
\hline 0007 & & {$[(($ lacht $))]$} \\
\hline 0008 & $\mathrm{H}$ & [((lacht)) das ist vielleicht vergleichbar ja] \\
\hline 0009 & I & [ja \\
\hline 0010 & S & ja] \\
\hline 0011 & & $\begin{array}{l}\text { und wie bei den erwachsenen gibts bei euch auch irgendwelche formen von } \\
\text { medikamentöser therapie was sind denn da so die unterschiede }\end{array}$ \\
\hline
\end{tabular}

Die Vergleiche in den Zeilen 06 und 11 beziehen sich auf das Fach- und Arbeitsgebiet der drei Hosts, nämlich die Erwachsenenpsychiatrie. An der zweiten Stelle nimmt Sebastian die Parallele, die Moritz zuvor gezogen hat, auf und leitet damit zur nächsten Frage an Herr über. Erneut lassen die Hosts ihre eigene 
Expertise einfließen, überlassen aber gleichzeitig Herr die Rolle des Interviewten und (Haupt-)Experten, der insgesamt den größten Redeanteil hat. Die vier Gesprächspartner:innen wären durchaus fähig, ein Fachgespräch zum Thema zu führen. Dies findet aber nicht statt, da der Podcast sich an ein breites Publikum richtet. ${ }^{6}$

Im Gegensatz zu den Beispielen aus dem Fernsehen unter Punkt 4.2 wechseln die Hosts nicht zwischen dem Status von Expert:innen und (scheinbaren) Lai:innen hin und her, sondern es findet eine Mischung der Rollen statt: Die Funktion der Moderation geht Hand in Hand mit der Anzeige des eigenen Wissensstatus. Ismene, Sebastian und Moritz nehmen eine intermediäre Rolle ein, die als Ko-Experte oder Ko-Expertin bezeichnet werden kann. Eine mögliche Erklärung für dieses Verhalten ist, dass sich der Podcast von klassischen Produktions- und Organisationsstrukturen des Rundfunks entfernt und daher die Hosts ein anderes Rollenverständnis erlangen (vgl. Jarrett, 2009; Dantas-Queiroz, Wentzel \& Queiroz, 2018). Zwar können auch Moderator:innen im Rundfunk die Rolle der Erklärenden übernehmen oder sogar Fachexpert:innen sein, wie die Beispiele aus den vorigen Abschnitten gezeigt haben. Allerdings bleibt ihre primäre Rolle die des Fragenden und der Vertretung des Publikums, was sogar dazu führen kann, dass sie ihr Wissen zurückhalten und vorgeben, weniger Kenntnisse zu haben, als es tatsächlich der Fall ist (s. Punkt 4.2). Im Podcast Jung und Freudlos ist diese Konvention schwächer ausgeprägt: Die Hosts führen das Gespräch auf allgemeinverständlichem Niveau, damit Zuhörende ohne Vorbildung folgen können, gleichzeitig verstehen sie sich als Vertreter:innen ihres eigenen Fachs.

Eine andere Ausprägung der Rolle des Ko-Experten zeigt sich bei Tim Schmidt (T) in seinem Podcast Transformatorische Bildung. Schmidt ist Bildungswissenschaftler und an der Universität Köln als Dozent tätig. ${ }^{7}$ In der Podcast-Folge (Schmidt 2019) hat er die Studentin Sirin (S) eingeladen, die im Rahmen ihres Studiums ein Forschungsprojekt umgesetzt hat:

\begin{tabular}{|c|c|c|}
\hline 0001 & $\mathrm{~T}$ & $\begin{array}{l}\text { genau das erste was ich immer ganz wichtig finde ist dass man für sich sozusagen } \\
\text { rausfindet was will man eigentlich wissen }\end{array}$ \\
\hline 0002 & $S$ & [mhm] \\
\hline 0003 & & {$\left[{ }^{\circ} \mathrm{h}\right]$} \\
\hline 0004 & $\mathrm{~T}$ & $\begin{array}{l}\text { das schöne im äh dieses forschungsprojekt im praxissemester das wird ja unter } \\
\text { forschendem lernen sozusagen diskutiert }\end{array}$ \\
\hline 0005 & & $\begin{array}{l}\text { `h da geht es jetzt nicht darum noch ne wissenschaftliche arbeit zu machen sondern es } \\
\text { geht vor allen dingen darum dass die studierenden die möglichkeit bekommen das was sie } \\
\text { sozusagen theoretisch an der uni sich in verschiedensten bereichen sozusagen }{ }^{\circ} \mathrm{h} \text { mal } \\
\text { gelernt haben }\end{array}$ \\
\hline 0006 & & (0.57) \\
\hline
\end{tabular}

6 https://juf.podigee.io/ (Zugriff am 01.11.2019).

7 https://transformatorische-bildung.de/ueber-mich/ (Zugriff am 01.11.2019). 


\begin{tabular}{|c|c|c|}
\hline 0007 & & $\begin{array}{l}\text { auf einen entsprechenden gegenstand also in diesem fall jetzt narrative interviews } \\
\text { entsprechend äh anzuwenden }{ }^{\circ} \mathrm{h}\end{array}$ \\
\hline 0008 & & (0.4) \\
\hline 0009 & & wieso war das für dich wichtig \\
\hline 0010 & S & also ich find das is an sich wirklich \\
\hline 0011 & $\mathrm{~T}$ & $\mathrm{hm}$ \\
\hline 0012 & $\mathrm{~S}$ & wirklich ne gute sache weil \\
\hline 0013 & & $\mathrm{hm}$ \\
\hline 0014 & & wir sind's einfach gewohnt hausarbeiten zu schreiben \\
\hline 0015 & & {$\left[{ }^{\circ} \mathrm{hh}\right]$} \\
\hline 0016 & $\mathrm{~T}$ & {$[\mathrm{hm}]$} \\
\hline 0017 & $\mathrm{~S}$ & $\begin{array}{l}\text { quellen äh zu untersuchen sekundär sich mit sekundärliteratur auseinanderzusetzen }{ }^{\circ} \mathrm{h} \\
\text { aber so wirklich ma }\end{array}$ \\
\hline 0018 & & $(1.43)$ \\
\hline 0019 & & ja \\
\hline 0020 & & [forschen] \\
\hline 0021 & $\mathrm{~T}$ & {$[\mathrm{hm}]$} \\
\hline 0022 & S & des lernen \\
\hline 0023 & & [zu betreiben] \\
\hline 0024 & $\mathrm{~T}$ & {$[\mathrm{hm}]$} \\
\hline 0025 & S & das kenn wir ein \\
\hline 0026 & & [fach nicht] \\
\hline 0027 & $\mathrm{~T}$ & {$[\mathrm{hm}]$} \\
\hline
\end{tabular}

Obwohl Sirin der Gast ist und sich die Folge auf ihre Arbeit bezieht, äußert sich zunächst Schmidt sehr ausführlich zum Zweck solcher Forschungsprojekte als Teil des Studiums. Die Frage an sie formuliert er erst sehr spät in Zeile 09. Sie nimmt daraufhin vieles auf, was er in seiner Vorrede bereits erwähnt hat, beispielsweise den Begriff des forschenden Lernens. Ab Zeile 14 paraphrasiert sie weitgehend, was Schmidt in Zeile 04 gesagt hat. Er tritt in der Gesprächsführung deutlich stärker in den Vordergrund und hat in diesem Abschnitt so- 
gar einen größeren Redeanteil als sein Gast, was im Vergleich zu dem vorherigen Ausschnitt aus der Folge von Jung und Freudlos (Hermann et al. 2019) besonders auffällt. Sirin kommt dann im späteren Teil der Folge (Schmidt 2019), der hier nicht abgebildet ist, die Rolle zu, ihre Erfahrungen und Erkenntnisse aus dem Projekt zu schildern.

Dozent und Studentin treten also beide als Expert:innen auf. Schmidt orientiert sich weniger am Turn-Design eines Nachrichteninterviews als die Hosts von Jung und Freudlos. Trotzdem gibt er dieses Muster nicht gänzlich auf, denn schließlich richtet er doch eine Frage an seinen Gast. Da Sirins Projekt im Mittelpunkt steht und Schmidt den Rahmen dazu schafft, ist es berechtigt, ihn ebenfalls als Ko-Experten zu bezeichnen. Statt Ergänzungen liefert er eine Vorlage für seine Gesprächspartnerin. Dennoch bleibt erkennbar, dass er derjenige ist, der sie interviewt und nicht umgekehrt.

\subsection{Umgang mit Unsicherheit bei Expert:innen und Lai:innen}

Eine dritte Gruppe von Akteur:innen in Wissenschaftspodcasts, die bisher nicht betrachtet wurde, sind die wissenschaftlichen Lai:innen. Wie unter Punkt 2 bereits erwähnt, haben Salvati (2015) und Barrios-O'Neill (2018) an einzelnen Podcasts aufgezeigt, wie Lai:innen mit ihrer Rolle als Hosts umgehen können: Sie thematisieren offen, dass sie über keinen fachwissenschaftlichen Hintergrund haben und distanzieren sich damit von der Rolle des wissenschaftlichen Experten bzw. Expertin (Salvati, 2015). Stattdessen bieten sie persönliche Interpretationen der präsentierten Inhalte aus der Forschung an und versuchen, interdisziplinäre herzustellen (ebd.). Barrios-O'Neill (2018) vergleicht sie mit Bürgerwissenschaftler:innen, die sich im Rahmen des Podcasts mit Wissenschaft beschäftigen und dadurch eigene Kenntnisse aufbauen.

Ebenso beachtenswert wie der Umgang mit Wissen ist der mit Nichtwissen und Unsicherheit. Im Podcast Ach $?^{8}$ sprechen die drei Hosts Jürgen (J), Philipp (P) und Dominik über historische Ereignisse, in der ausgewählten Folge geht es um die erste Tour de France im Jahr 1903 (Krauß et al. 2019). Es ist für die Zuhörenden ersichtlich, dass keiner der drei Hosts Historiker ist oder einen anderen fachlichen Bezug zum Feld hat. Vielmehr übernimmt Jürgen die Rolle des Experten, da er die Quellen zum Thema recherchiert hat und die Ergebnisse seinen Gesprächspartnern in Form einer Erzählung vorträgt. Dabei diskutieren sie ein technisches Detail:

0001 J $\begin{aligned} & \text { und des ist jetzt auch net so dass die die technik der vorderradbremsen jetzt schon } \\ & \text { irgendwie high end wär ne also die ham so ganz klobige irgendwie bremsen die auf } \\ & \text { holzfelgen sowieso nur so halb geil funktionieren }\end{aligned}$
0002
[ja]
[aber mal]
$\frac{8 \text { https://das-a.ch/ (Zugriff am 01.11.2019). }}{\text { P }}$




\begin{tabular}{|c|c|c|}
\hline 0004 & & ganz kurz noch gefragt \\
\hline 0005 & & n wo drücken die bremsklötze dagegen \\
\hline 0006 & $\mathrm{~J}$ & das kann ich dir net sagen aber \\
\hline 0007 & & [ich glaub dass] \\
\hline 0008 & $\mathrm{P}$ & [ich hatte nämlich] \\
\hline 0009 & $\mathrm{~J}$ & dir das \\
\hline 0010 & & [schon] \\
\hline 0011 & $\mathrm{P}$ & [ja] \\
\hline 0012 & $\mathrm{~J}$ & halbwegs so vorstellen kannst wie heute die ham \\
\hline 0013 & & [ähm] \\
\hline 0014 & $\mathrm{P}$ & [ja] \\
\hline 0015 & $\mathrm{~J}$ & pneureifen mit \\
\hline 0016 & & [mantel und] \\
\hline 0017 & $\mathrm{P}$ & {$[\mathrm{mhm}]$} \\
\hline 0018 & $\mathrm{~J}$ & schlauch ja ${ }^{\circ} \mathrm{h}$ und ähm halt auf felgen die womöglich in den meisten fällen aus holz sind \\
\hline 0019 & $\mathrm{P}$ & ich hatte nämlich $\mathrm{n}$ fahrrad das war wirklich wirklich alt \\
\hline 0020 & & $(0.54)$ \\
\hline 0021 & & und das hatte eine sensationelle bremse ${ }^{\circ} \mathrm{h}$ \\
\hline
\end{tabular}

Im Gegensatz zu den bisher vorgestellten Podcast-Folgen tritt hier kein Gast auf. Jürgen wird daher von den anderen beiden Hosts auch nicht befragt, vielmehr trägt er die vorbereitete Geschichte vor, während Philipp und Dominik gelegentlich Zwischenfragen stellen oder das Gesagte kommentieren. In diesem Abschnitt räumt Jürgen ein, dass er die Funktionsweise der damaligen Bremsen nicht im Detail recherchiert hat. Da es um ein Thema geht, dass sehr nah am Alltagswissen liegt, vermutet er ein ähnliches Prinzip wie bei heutigen Fahrrädern. Einer seiner Gesprächspartner nimmt den Faden auf und an den Abschnitt schließt sich eine Unterhaltung der drei Hosts über eigene Fahrräder an.

Die Art, wie die Podcaster mit der Wissenslücke umgehen, erinnert an die Beschreibung von Debatten mit Lai:innen im Fernsehen bei Livingstone \& Lunt (1994). Sie charakterisieren die Meinungen, die Lai:innen aufgrund ihrer Er- 
fahrungen einbringen u.a. als subjektiv, konkret, auf Vermutungen begründet, (persönlich) motiviert und offensichtlich/intuitiv. Die Beiträge von Expert:innen zeichneten sich im Kontrast dazu als objektiv, abstrakt, faktisch begründet, neutral und kontra-intuitiv aus (Livingstone \& Lunt, 1994, S. 102). Die Charakteristika des Laiendiskurses finden sich in dem Ausschnitt der Ach?Folge (Krauß et al. 2019) wieder.

Einen Vergleich bietet der Podcast Sternbildung ${ }^{9}$, den Holger Klein und Florian Freistetter im Auftrag des Verlags Spektrum der Wissenschaft produziert haben. Wie bei Ach? gibt es keine Gäste, allerdings tritt Freistetter (F) als Astronom auf und verfügt daher im Gegensatz zu Klein (K) über explizites Fachwissen. Thema der Folge (Freistetter/Klein 2019) ist eine bestimmte Klasse von Himmelskörpern, sogenannte Braune Zwerge, über die Freistetter erzählt. Es folgt ein Einwand von Klein, der sich ebenfalls auf unsicheres Wissen bezieht:

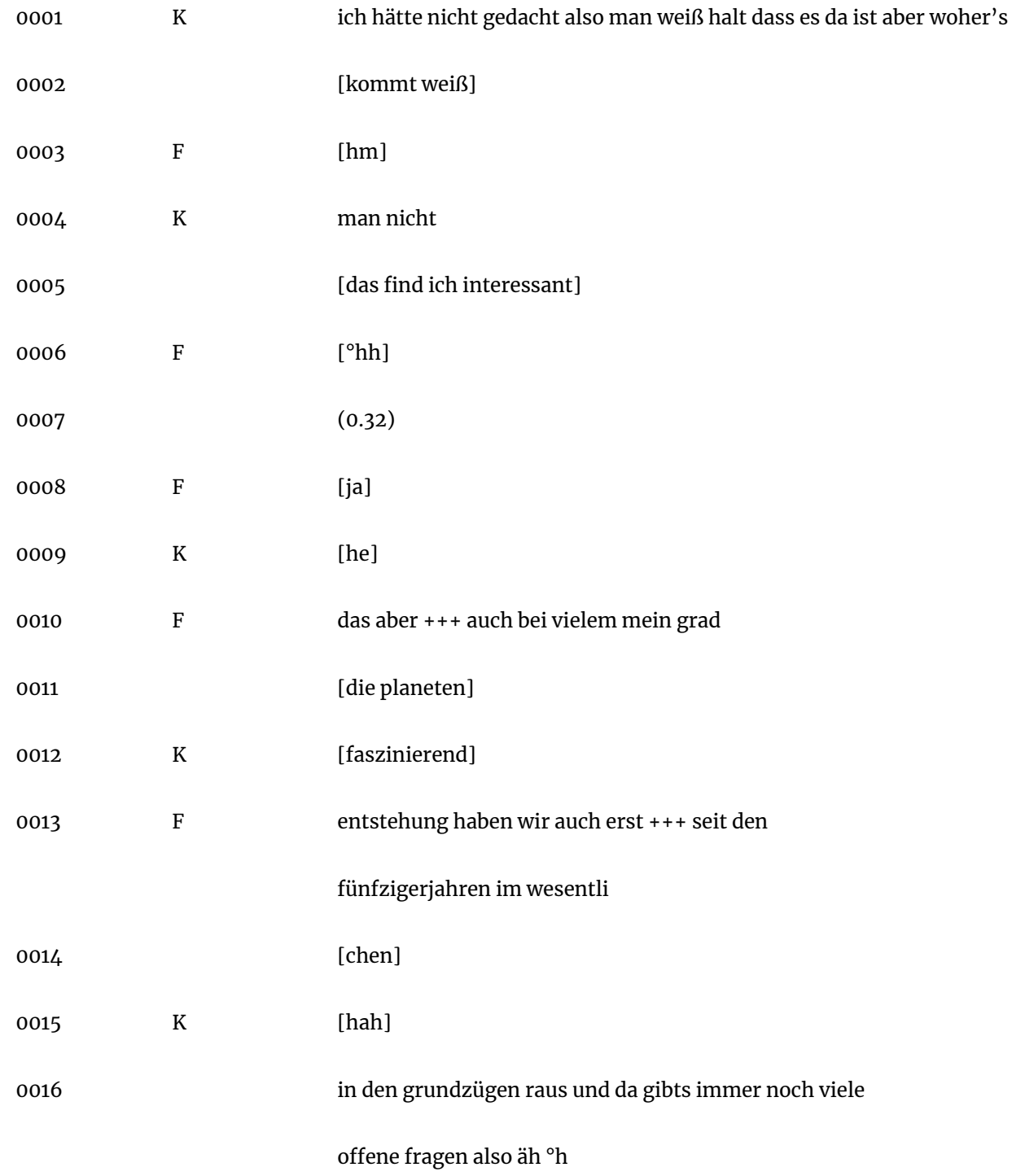

0016

[kommt weiß]

[hm]

man nicht

[das find ich interessant]

$\left[{ }^{\circ} \mathrm{hh}\right]$

$(0.32)$

[ja]

[he]

das aber +++ auch bei vielem mein grad

[die planeten]

[faszinierend]

entstehung haben wir auch erst +++ seit den

fünfzigerjahren im wesentli

[chen]

[hah]

in den grundzügen raus und da gibts immer noch viele

offene fragen also äh ${ }^{\circ} \mathrm{h}$

9 https://scilogs.spektrum.de/sternbildung/ (Zugriff am 01.11.2019). 
0017 vor allem äh das ding is ja dass wir die braunen

zwergen ja auch noch nicht so lange kennen
Dieser kurze Diskurs ist von anderer Natur als im vorigen Beispiel, was einerseits an der Wahl des Themas liegen mag, das kaum Bezug zum Alltagswissen zulässt. Andererseits ist die Konstellation der Gesprächspartner entscheidend. Freistetter bleibt auf der fachlichen Ebene und stellt die Frage nach der Herkunft der Braunen Zwerge in Zusammenhang mit dem Erkenntnisfortschritt in der Wissenschaft (vgl. Rhein, 2015, S. 357ff). Durch den Bezug zur Planetenentstehung ab Zeile 11 stellt er dar, dass es sich bei den Braunen Zwergen um ein „Noch-nicht-Wissen“ (ebd., S. 383) handelt, das durch weitere Forschung zumindest teilweise überwunden werden könnte. Ähnliche Argumentationen konnte Rhein bei interdisziplinären wissenschaftlichen Diskussionen nachweisen (ebd.). Das erklärt, warum Klein sich nicht in Freistetters Erörterung einschaltet, abgesehen von dem nachgeschobenen „faszinierend“ in Zeile 12. Er hat mit seiner einleitenden Bemerkung zwar den Anstoß gegeben, überlässt aber Freistetter die Erläuterung. Er erkennt Freistetter damit die Deutungshoheit zu, wenn es um Grundverständnisse der Wissenschaft geht. Sowohl Klein als auch Freistetter bleiben daher in ihren Rollen - der des Laien bezogen auf das Thema Astronomie und der des wissenschaftlichen Fachmanns.

In beiden Fällen hat ein Laie eine Nachfrage an den Experten zum Thema gestellt. Wie die Frage beantwortet wird und wer an der Beantwortung teilnimmt, hängt davon ab, über welche Art von Expertise das Gegenüber verfügt. Mit diesem Vergleich wird das Konzept des Ach?-Podcast noch klarer: Da keiner der Hosts über einen fachlichen Hintergrund verfügt, diskutieren sie alle drei auf der gleichen Ebene. Wie bei Salvati (2015) und Barrios-O'Neill (2018) ist es ein besonderes Merkmal solcher Podcasts, dass wissenschaftliche Inhalte aus Sicht von Lai:innen verhandelt und dargestellt werden. Die Beobachtung deckt sich zudem mit der Analyse von Birch und Weitkamp, die Diskussionen zu einzelnen Folgen von Wissenschaftspodcasts in Kommentarspalten betrachtet haben: In einem Fall schaltete sich ein Hosts ein, der über fachliche Expertise zum Thema verfügte. Die übrigen Nutzenden hielten sich mit ihren Kommentaren stärker zurück als bei anderen Diskussionen, an denen keine Person aus dem Fach teilnahm (Birch \& Weitkamp, 2010). In schriftlich geführten Diskursen wurde ein ähnlicher Fall also schon beschrieben - wie sich das Expert:in/Lai:in-Verhältnis im konkreten Gesprächsverhalten ausprägt, wurde hiermit exemplarisch demonstriert.

\section{Fazit}

Bereits in den wenigen Ausschnitten konnte beispielhaft gezeigt werden, wie Hosts von Wissenschaftspodcasts ihre Rollen interpretieren und ausgestalten. 
Dabei treten viele Ähnlichkeiten zu Gesprächen z.B. im institutionellen Rundfunk oder freien Radio auf. Da Podcasts jünger sind als die anderen genannten Medien, war zu erwarten, dass darin zahlreiche Einflüsse zusammenkommen. Abweichende Interpretationen von Rollen entstehen hingegen durch neue Konstellationen von Akteur:innen, die durch die Bedingungen des Mediums Podcast in Interaktion treten. In den Beispielen traten Fachexpert:innen mit anderen Fachexpert:innen auf (Jung und Freudlos, Transformatorische Bildung), Fachexpert:innen gemeinsam mit Lai:innen (Sternbildung, THINK \& DO) sowie Lai:innen mit anderen Lai:innen (Ach?). In jeder Konstellation haben die Akteur:innen auf andere Art und Weise an der Diskussion über wissenschaftliche Themen teil. Ebenfalls zu beobachten ist, dass die Hosts der Podcasts typische Aufgaben der Moderation übernehmen, die eigene Position (als Pressesprecher:in, Arzt/Ärztin, Dozent:in, ...) jedoch häufig in den Vordergrund rückt.

Die Ergebnisse beziehen sich nur auf ein bestimmtes Beitragsformat von Podcasts - dem Gespräch - und da sie lediglich auf dem Minimaltranskript basieren, ist die Analyse noch relativ oberflächlich. Dennoch lässt sich aus den Ausschnitten mehr herauslesen, als es durch die Auswertung von Meta-Daten und Beschreibung der Podcast-Folgen möglich gewesen wäre. Wenn man lediglich Informationen wie Themen, Produzierende etc. ausgewertet, wie es bei anderen Studien der Fall ist, wären die Dynamiken, die zwischen den Akteur:innen im direkten Austausch entstehen, verborgen geblieben. Auf der anderen Seite stehen Arbeiten, die einzelne Aspekte anhand von einem oder wenigen Fallbeispielen erörtern. Doch gerade der Vergleich mehrerer Fälle unter verschiedenen Gesichtspunkten - obschon auch hier das Sample klein ist - kann vielfältige Einsichten hervorbringen.

Aus der Praxis der Wissenschaftskommunikation äußern sich insbesondere Forschende zum Medium Podcast, das sie dazu nutzen, um über ihre Arbeit zu sprechen. Der Austausch mit anderen Gesprächspartner:innen ermögliche es ihnen dabei, ihre Themen ausführlich darzustellen und selbst als Personen hinter ihrer Arbeit sichtbar zu werden (Grasberger, 2016; Hu, 2016; Sack, 2017). Mithilfe der Gesprächsanalyse können solche bisher subjektiven Eindrücke auf empirischer Basis geprüft und diskutiert werden. Gleichzeitig wurde in diesem Beitrag der Versuch unternommen, weitere Akteur:innen wie beispielsweise PR-Verantwortliche als Hosts von Podcasts in den Fokus zu rücken, die in der Forschungsliteratur noch wenig Beachtung finden. Bei näherer Betrachtung jeder einzelnen Gruppe von Akteur:innen würden sich sicher weitere interessante Fragestellungen ergeben, die mit Methoden der Gesprächsanalyse beantwortet werden können. Das dafür benötigte Material ist optimal zugänglich, da Podcast-Folgen bereits als Dateien zum Download vorliegen und in der Regel über lange Zeiträume dauerhaft zugänglich sind. Die Aufnahmen der Gespräche müssen nicht erst angefertigt oder auf anderen Wegen beschafft werden.

Podcasts stellen sich daher insgesamt nicht nur als lohnendes Feld für die Praxis der Wissenschaftskommunikation dar, sondern auch für deren Erfor- 
schung. Die Methode der Gesprächsanalyse beweist sich als geeignet für die vorgestellte Fragestellung, daher werden sich in der weiteren, vertiefenden Analyse hoffentlich weitere interessante Phänomene beschreiben lassen.

\section{Literatur}

Barrios-O'Neill, D. (2018). Wild Listening: Ecology of a Science Podcast. In D. Llinares, N. Fox \& R. Berry (Hrsg.), New aural cultures and digital media (1st edition., S. 147-172). New York, NY: Springer Science+Business Media, LLC.

Birch, H. \& Weitkamp, E. (2010). Podologues: conversations created by science podcasts. new media \& society, 12(6), 889-909. https://doi.org/10.1177/ 1461444809356333

Bottomley, A. J. (2015). Podcasting, welcome to night vale, and the revival of radio drama. Journal of Radio \& Audio Media, 22(2), 179-189. https://doi.org/10.1080/19376529.2015.1083370

Bruce, B. (. \& Lin, C.-C. (2009). Voices of youth: Podcasting as a means for inquiry-based community engagement. E-Learning and Digital Media, 6(2), 230-241. https://doi.org/10.2304/elea.2009.6.2.230

Burger, H. (1991). Das Gespräch in den Massenmedien. Berlin: de Gruyter.

Burger, H. \& Luginbühl, M. (2014). Mediensprache: eine Einführung in Sprache und Kommunikationsformen der Massenmedien (4., neu bearbeitete und erweiterte Auflage.). Berlin ; Boston: De Gruyter.

Chamberlin, F. (2019). Podcasting historias: Public outreach through digital storytelling in iberian history. Bulletin for Spanish and Portuguese Historical Studies, 43(1), 104-133. https://doi.org/10.26431/0739-182X.1291

Chovanec, J. (2016). "It's quite simple, really": Shifting forms of expertise in TV documentaries. Discourse, Context \& Media, 13, 11-19. https://doi.org/10.1016/j.dcm.2016.03.004

Clayman, S. E. (o. J.). Conversation Analysis in the News Interview. In The Handbook of Conversation Analysis (S. 630-656). John Wiley \& Sons, Ltd. https://doi.org/https://doi.org/10.1002/9781118325001.ch31

Dantas-Queiroz, M. V., Wentzel, L. C. P. \& Queiroz, L. L. (2018). Science communication podcasting in Brazil: the potential and challenges depicted by two podcasts. Anais da Academia Brasileira de Ciências, 9o(2), 1891-1901. https://doi.org/10.1590/0001-3765201820170431

Deck, R. \& Kunow, K. (2019). Webradio, Musikstreaming, Podcast und Smart Speaker - Die Vermessung von Audio-Neuland. Ergebnisse des Online-Audio-Monitors 2019. München. Verfügbar unter: https://www.online-audio-monitor.de/wpcontent/uploads/OAM-aus-DigiBericht_2019_AUDIO_final_WEB.pdf 
Deppermann, A. (2008). Gespräche analysieren: Eine Einführung. Wiesbaden: VS Verlag für Sozialwissenschaften / GWV Fachverlage GmbH, Wiesbaden.

Deppermann, A. (2018). Wissen im Gespräch (Handbücher Sprachwissen (HSW)). In K. Birkner \& N. Janich (Hrsg.), Handbuch Text und Gespräch (S. 104-142). Boston: De Gruyter Mouton.

Domenichini, B. (2018). Podcastnutzung in Deutschland. Media Perspektiven, (2), 46-49.

Goffman, E. (1981). Forms of talk (University of Pennsylvania publications in conduct and communication). Philadelphia: University of Pennsylvania Press.

Grasberger, L. (2016, August 19). Der Ton macht die Wissenschaft. MERTON Magazin. Verfügbar unter: https://merton-magazin.de/der-ton-macht-diewissenschaft

Hellermann, M. (2015). Wissenschaft in Film und Fernsehen: die mediale Morphologie audiovisueller Wissenschaftskommunikation (Schriften zur Medienmorphologie und Medienphilosophie). Berlin: LIT Verlag.

Henne, H. \& Rehbock, H. (1979). Einführung in die Gesprächsanalyse (Sammlung Göschen ; 2212). Berlin ; New York: de Gruyter.

Heritage, J. \& Clayman, S. (2010). Talk in action: interactions, identities, and institutions (Language in society). Chichester Malden: Wiley-Blackwell.

$\mathrm{Hu}$, J. C. (2016). Scientist ride the podcasting waves. sciencemag.org. Verfügbar unter: http://www.sciencemag.org/careers/2016/11/scientists-ridepodcasting-wave

Hutchby, I. (2007). Media Talk: Conversation Analysis and the Study of Broadcasting. Maidenhead: McGraw-Hill International (UK) Ltd.

Hutchby, I. \& Wooffitt, R. (2008). Conversation analysis (2nd ed.). Cambridge, UK ; Malden, MA: Polity.

Jarrett, K. (2009). PRIVATE TALK IN THE PUBLIC SPHERE. Communication, Politics and Culture, (42), 116-135.

Kitzinger, C. (2012). Repair. In J. Sidnell \& T. Stivers (Hrsg.), The Handbook of Conversation Analysis: Sidnell/The Handbook of Conversation Analysis (S. 229-256). Chichester, UK: John Wiley \& Sons, Ltd. https://doi.org/10.1002/ 9781118325001

Livingstone, S. M. \& Lunt, P. K. (1994). Talk on television: audience participation and public debate. London; New York: Routledge. Zugriff am 16.3.2020. Verfügbar unter: http://www.dawsonera.com/depp/reader/protected/external/ AbstractView/S9780203131916

Lüders, M. (2008). Conceptualizing personal media. new media \& society, Vol. 10(05), 683-702. 
MacKenzie, L. E. (2019). Science podcasts: Analysis of global production and output from 2004 to 2018. Royal Society Open Science, 6(1), 180932. https://doi.org/10.1098/rsos.180932

Markman, K. M. (2012). Doing radio, making friends, and having fun: Exploring the motivations of independent audio podcasters. New Media \& Society, 14(4), 547-565. https://doi.org/10.1177/1461444811420848

Markman, K. M. \& Sawyer, C. E. (2014). Why pod? Further explorations of the motivations for independent podcasting. Journal of Radio \& Audio Media, 21(1), 20-35. https://doi.org/10.1080/19376529.2014.891211

Martens, D. \& Amann, R. (2007). Podcast: Wear-out oder Habitualisierung? Media Perspektiven, (11), 538-551.

Norman, K. (2018, Juni). Listening In: The Work We Do Now - A Conversation Analysis of Power Dynamics Between Podcast Hosts. Honors Senior Theses/Projects. Monmouth: Western Oregon University.

Nölleke, D. (2013). Experten im Journalismus: systemtheoretischer Entwurf und empirische Bestandsaufnahme (Schriftenreihe "Aktuell. Studien zum Journalismus") (1. Auflage.). Baden-Baden: Nomos.

Peters, H. P. (1994). Wissenschaftliche Experten in der öffentlichen Kommunikation über Technik, Umwelt und Risiken. Kölner Zeitschrift für Soziologie und Sozialpsychologie, (46), 162-190.

Pinseler, J. (2001). Sprechen im freien Radio - Eine Fallanalyse zu Möglichkeiten alternativen Hörfunks. Medien \& Kommunikationswissenschaft, Jahrgang 49(Heft 3).

Rhein, L. (2015). Selbstdarstellung in der Wissenschaft: eine linguistische Untersuchung zum Diskussionsverhalten von Wissenschaftlern in interdisziplinären Kontexten (Wissen, Kompetenz, Text). Frankfurt am Main: Peter Lang Edition.

Sack, G. (2017, März 17). Let's talk about science podcasts. berkeley science review. Verfügbar unter: http://berkeleysciencereview.com/lets-talk-aboutscience-podcasts/

Salvati, A. J. (2015). Podcasting the past: Hardcore history , fandom, and DIY histories. Journal of Radio \& Audio Media, 22(2), 231-239. https://doi.org/10.1080/19376529.2015.1083375

Schaffer, T. (2017, Juli 11). Wer hört Podcast in Österreich? Die Ergebnisse unserer Hörerumfrage. zurPolitik.com. Verfügbar unter: http://zurpolitik.com/ 2016/07/11/podcasts- oesterreich-umfrage-2016/

Schäfer, M. S., Kristiansen, S. \& Bonfadelli, H. (Hrsg.). (2015). Wissenschaftskommunikation im Wandel: 1. Jahrestagung der Ad-hoc-Gruppe "Wissenschaftskommunikation" der DGPuK, die im Januar 2014 an der Universität Zürich stattgefunden hat. Köln: von Halem. 
Selting, M., Auer, P. \& Barth-Weingarten, D. et a. (2009). Gesprächsanalytisches Transkriptionssystem 2 (GAT 2). Gesprächsforschung - Online-Zeitschrift zur verbalen Interaktion, (10), 353-402.

Swiatek, L. (2018). The Podcast as and Intimate Bridging Medium. In D. Llinares, N. Fox \& R. Berry (Hrsg.), New aural cultures and digital media (1st edition., S. 173-187). New York, NY: Springer Science+Business Media, LLC.

Teckchandani, A. \& Obstfeld, D. (2017). Storytelling at its best: Using the StartUp podcast in the classroom. Management Teaching Review, 2(1), 26-34. https://doi.org/10.1177/2379298116674696

Tynan, B. \& Colbran, S. (2006). Podcasting, student learning and expectations. In ASCILITE 2006: Who's Learning? Whose Technology? (S. 825-832). Verfügbar unter: http://oro.open.ac.uk/37970/

Welbourne, D. J. \& Grant, W. J. (2016). Science communication on YouTube: Factors that affect channel and video popularity. Public Understanding of Science, 25(6), 706-718. https://doi.org/10.1177/0963662515572068 